\title{
L'habiter multilocal : discussion d'un concept émergent et aperçu de sa traduction empirique en Suisse
}

\author{
CÉDRIC DUCHÊNE-LACROIX \\ NICOLA HILTI ${ }^{2}$ \\ HELMUT SCHAD ${ }^{3}$
}

\begin{abstract}
Résumé
L'habiter multilocal comme pratiques à la fois d'ancrages locaux et de circularité entre lieux d'habitation géographiquement éclatés bouscule le paradigme habituel du double container territorial, celui de la gestion des territoires administratifs et celui de l'adéquation entre une personne, son ménage et son logement d'appartenance unique. L'habiter multilocal est un ensemble de modes de vie mobilo-sédentaires hybrides (living apart together; enfant pendulaire entre parents séparés, etc.) pour partie émergents et constitue un nouveau champ de recherche. Pour y voir plus clair, l'article propose tout d'abord un cheminement conceptuel entre habitat, pratique et pratiquant au plus près des enquêtes suisses quantitatives existantes. II s'ensuit une analyse critique de données existantes sur la birésidentialité formelle et ce qu'elles peuvent nous apporter pour comprendre l'ensemble du phénomène de l'habiter multilocal. Au vu des premiers résultats, l'habiter multilocal est une pratique non négligeable en Suisse, marquée par les structures sociales, mais demande à être mieux éclairé démographiquement et sociologiquement, audelà même de l'actualité du trop-plein controversé de résidences secondaires dans les régions touristiques. L'article suggère quelques pistes de recherche concrètes provenant des réflexions autour du projet de recherche "L'habiter multilocal en Suisse».
\end{abstract}

\section{Mots-clés}

Habiter multilocal, multirésidentialité, résidences secondaires, Suisse, Étude de la multilocalité résidentielle.

\footnotetext{
1. Université de Bâle.

2. ETH Zürich.

3. Hochschule Luzern.
} 


\begin{abstract}
Summary
Multilocal dwelling as both local anchoring practices and circularities between dispersed living places rushes the usual double territorial container paradigm: the container of territorial administrative management and the container matching each person in one household in one housing together. Multilocal dwelling is a set of mobile-settled hybrid ways of life (living apart together; commuting children between separated parents, etc.) which are partly emerging and constitute a new field of research. To get a clearer picture of this new field the authors propose first of all a conceptual path through housing, multilocal practitioners and practices as close as possible to the existing quantitative Swiss surveys. Then they analyze the available data on formal bi-residentiality and what they provide to better understand the whole multilocal dwelling phenomenon. In light of these first results multilocal dwelling practices in Switzerland are significant and social structural coined but require to be demographically and sociologically better studied even beyond the current controversy about the overflow of second homes in the tourist areas. The authors suggest some implementing ideas from reflections linked to the research project «Multilocal dwelling in Switzerland».
\end{abstract}

\title{
Keywords
}

Multilocal dwelling, multi-residentiality, second homes, Switzerland, Residential multilocality studies.

\section{Introduction}

L'opposition franche entre sédentarité et nomadisme a vécu. Les habitants des pays développés, mieux pourvus économiquement, techniquement et en savoir-circuler, ont accès à des moyens de transports et de communication plus rapides - relevant les attentes de performance de mobilité - et se faisant à des lieux «lointains» d'habitation pour le travail, les loisirs, l'entourage, etc. Pour autant les pratiques restent localement sédentaires, attachées à des structures territoriales (école, foyer fiscal, ménage familial, présence sur le lieu de travail, etc.). Prises dans des contraintes et des opportunités d'ancrages ${ }^{4}$ localisés et de mobilités pour y accéder, les personnes vivent à leur échelle un éclatement géographique des lieux de vie pratiqués. Ces lieux sont ceux du quotidien (mobilité journalière, pendulaire, virtuelle), des déménagements (migrations infra- ou internationales), mais aussi - encore peu aperçus -

4. Le terme d'ancrage permet de dépasser l'opposition entre mobilité et sédentarité en rassemblant à la fois le lien au lieu et le potentiel de mobilité du bateau entre des îlots d'archipel (Duchêne-Lacroix, 2007, 2011a ; Sencébé, 2007). 
des lieux simultanés d'habitation (habiter multilocal). L'habiter multilocal, est l'ensemble des pratiques habituelles formelles (par exemple, résidences principale et secondaire) et informelles (par exemple, chez un ami et chez ses parents) d'habitation en plusieurs lieux distincts. Par rapport aux définitions existantes (Hilti, 2009, 2012 ; Weichhart, 2009 ; Petzold et al., 2010), nous insistons aussi sur le caractère informel de la pratique qui, nous allons le voir, est un défi pour l'enregistrement statistique de l'ensemble du phénomène. L'habiter multilocal produit et est le produit de la «mobilité hybride» (Vincent-Geslin, Kaufmann, 2012) entre circularité quotidienne et migration résidentielle (Weichhart, 2009), mais aussi de la sédentarité hybride. L'habiter multilocal peut s'apparenter à du nomadisme, car il y a déplacement circulaire entre lieux d'habitation, mais aussi à de la sédentarité car ces lieux de vie sont appropriés par habitude, par des symboles, des objets matériels et très souvent légalement par bail ou titre de propriété. Nous adhérons de ce fait au postulat des représentants des «mobility studies» selon lequel il n'est plus possible de séparer l'habiter et la mobilité (Urry, 2000 ; Hannam et al., 2006).

Mieux connaître l'habiter multilocal est nécessaire aujourd'hui. C'est notamment un enjeu de politique locale et au-delà : usages et besoins particuliers dans les domaines du transport, de l'habitat (par exemple, type de logement, pression foncière, risques de «lits froids» ${ }^{5}$ (Kissling, 2010), des offres de services (gardiennage, horaire des commerces, etc.), de l'énergie (coût énergétique et pérennité de comportements); bienfaits locaux ou non de certaines populations mobiles (par exemple, classes «créatives» (Florida, 2005) ; intégration problématique ou non des «intermittents multilocaux» dans la cité). L'habiter multilocal est aussi un enjeu de cohésion avec l'entourage (chance d'entretenir des liens lointains, mais risque de perte de sociabilité locale). C'est un arrangement socio-matériel, qui d'un point de vue de la théorie pratique doit être construit et stabilisé en permanence (Schad, Duchêne-Lacroix, 2013). L'habiter multilocal mobilise au niveau individuel des ressources, des compétences et des rapports identitaires territoriaux (Duchêne-Lacroix, Schad, 2013). Enfin, de nombreuses formes d'habiter multilocal émergent et peuvent préfigurer de nouvelles formes standard de vie en société (Kesselring, 2006).

Les études sur le sujet sont naissantes. Dans le monde germanique, on peut considérer le numéro double de l'IZR «Multilokales Wohnen» publié en 2009 sur la base de travaux antérieurs à 2007 comme un mo-

5. Excès du nombre de logements secondaires peu habités. 
ment fondateur d'une recherche pluridisciplinaire trinationale sur le sujet (Sturm, Weiske, 2009). En France, l'ouvrage collectif «D'une maison l'autre» fait référence (Bonnin, Villanova, 1999). Par ailleurs, certaines individualités ont depuis plus longtemps travaillé le phénomène et les concepts s'y rattachant, notamment le «système résidentiel» (Dureau, 2002) ou la «résidence multilocal» (Ember, 1972). Les situations d'habiter multilocal sont nombreuses, mais leur lecture synoptique est à faire. Dans des études portant sur des populations ou des situations assez différentes se retrouve la question de l'habiter multilocal : la birésidentialité de villégiature infranationale et internationale (Bonnin, Villanova, 1999 ; Baerenholdt, 2007), les résidences secondaires des retraités (Pierre, 2006), les (enfants de) familles recomposées (Clément, Bonvalet, 2005 ; Schier, 2009), les navetteurs professionnels (Reuschke, 2010) et bien entendu les migrants transnationaux (Tarrius, 1992; Pries, 2001 ; Bryceson, Vuorela, 2002 ; Le Gall, 2005 ; Zoll, 2007 ; Arsenault, 2009; Diminescu, 2010) dont Miranda définit la multilocalité (1996) ou Duchene-Lacroix l'archipel (Duchêne-Lacroix, 2007, 2011a).

La Suisse tient sa place dans la recherche sur l'habiter multilocal avec des travaux pionniers tant théoriques qu'empiriques. Il y a d'une part un questionnement scientifique sur les résidences secondaires dans les Alpes (Perlik, 2011; Sonderegger, 2012) sur fond de controverse politique à propos des avantages et inconvénients de celles-ci. La polémique a connu une nouvelle étape récemment avec l'acceptation de l'initiative populaire sur la limitation des résidences secondaires (Fondation Franz Weber, Helvetia Nostra, 2011 ; Conseil fédéral, 2012). Avec les tensions sur le marché immobilier, les villes aussi questionnent leur niveau de résidences secondaires (Odermatt, 1990 ; Kissling, 2010 ; Duchêne-Lacroix, 2011b). Il y a d'autre part des travaux fondateurs sur l'habiter polytopique (Stock, 2006), le sens, la culture et les configurations de l'habiter multilocal (Rolshoven, 2004, 2006 ; Hilti, 2007, 2009, 2012). Enfin, le laboratoire Lasur de Lausanne poursuit des recherches sur les formes atypiques de mobilité par lesquelles il aborde aussi la multirésidentialité (Vincent-Geslin, Kaufmann, 2012).

Des enquêtes quantitatives sur l'habiter multilocal proprement dit sont très peu nombreuses. Elles ont été réalisées à des échelles régionales en Allemagne (Sturm, Meyer, 2009) ou pour la première fois à une échelle nationale en France dont l'exploitation va débuter en 2013 (enquête Famille et logements 2011, INSEE/INED). Dans 6 pays européens, dont la Suisse, l'enquête Jobmob a ouvert la voie à un questionnement no- 
tamment en Suisse sur les multirésidents 6 . On note en outre des exploitations statistiques descriptives sur les résidences secondaires en Suisse (Roth, Braun, 2005 ; ARE, 2009), mais qui ne représentent qu'une partie émergée statistiquement et visuellement - dans les villages - du phénomène de l'habiter multilocal.

C'est qu'il y a des obstacles méthodologiques et épistémologiques à l'analyse du phénomène (Duchêne-Lacroix, 2011b). L'habiter multilocal, pratiqué pourtant, ne fait pas partie des concepts établis dans la société. Il sort du paradigme habituel du double container territorial: celui, d'une part de la gestion administrative des territoires politiques du national (mutualisé dans Schengen) au communal et dans laquelle s'inscrivent les domiciles et celui, d'autre part, de l'assignation administrative de chaque habitant à un ménage correspondant à un domicile. C'est sur cette base domiciliaire formelle qu'on parle du reste de sédentarité, qui ne correspond pas toujours aux pratiques spatiales des habitants. L'habiter multilocal pose ainsi la question de ce qu'est le domicile, le ménage, l'habiter, la sédentarité, le seuil à partir duquel l'habiter devient multilocal (par exemple quelle durée minimale de séjour sur les lieux d'habitation?), mais aussi comment atteindre ces populations multilocales et quelle est l'aire d'enquête. Il y a un besoin d'éclaircissement au plus près des réalités empiriques. Cet article a ainsi, dans une première partie, pour but de cheminer conceptuellement sur la base critique des questionnements et des définitions apparentés dans les enquêtes officielles suisses.

En seconde partie, l'analyse des quelque bases de données suisses se rapportant au sujet, bien qu'imparfaites, peu nombreuses, décalées par rapport au questionnement général sur l'habiter multilocal, permet de donner quelques points de repère sur l'habiter multilocal comme usage d'un logement dit secondaire, mais surtout d'ouvrir des pistes de réflexion fructueuses pour améliorer la visibilité statistique de ce phénomène.

6. www.jobmob-and-famlives.eu. 


\section{De la hiérarchie résidentielle aux pratiques informelles}

\section{Des résidences secondaires aux birésidentiaux}

L'habiter multilocal centré sur la pratique, inclut l'usage de la résidence secondaire comme jouissance formelle de plus d'un logement en propre (location, propriété). Traditionnellement, la plupart des données disponibles sur les habitations hiérarchisent a priori des lieux d'habitation (résidence principale/résidence secondaire) (Bonnin, 1999). Mais les critères de distinction entre logement principal et secondaire ne sont pas unifiés. Ainsi, pour les résultats du Recensement Fédéral de la Population (RFP) suisse, les résidences secondaires sont les logements habités temporairement déduits des questionnaires remis aux propriétaires des logements. Pour les microrecensements mobilités et transports $(M R T)^{7}$ de 2005 et 2010 organisés aussi par l'Office de la Statistique Suisse (en collaboration avec l'office fédéral du développement territorial) et le Panel Suisse des Ménages (PSM, vague de 1999 et 2003), il s'agit des logements (de la maison à la chambre) dont les ménages résidant en Suisse disposent en propre (location ou propriété) en plus d'un logement principal. Sont exclus du champ pour le MRT comme pour le PSM, les logements en Suisse loués ou détenus par des non-résidants en Suisse. Une autre définition de la résidence secondaire est proposée par André Odermatt :

«Toutes les opportunités résidentielles, qui sont utilisées par la famille ou une personne de façon intermittente mais aussi souvent avec une certaine régularité pour l'hébergement et l'approvisionnement ménager, en plus de la résidence principale où se concentre la vie quotidienne de la famille ou d'une personne. Ces opportunités résidentielles peuvent être en propriété, en location ou bail de longue ou courte durée, ou mise à disposition» (1990, traduction des auteurs).

Cette définition élargit l'ensemble de la résidence secondaire à la résidentialité secondaire informelle, ce qui permet ainsi d'inclure les résidences plus précaires, par exemple les prêts amicaux ou les habitacles

7. Le microrecensement transports de 2005 est une enquête par échantillon sur 60000 ménages et autant de membres des ménages sélectionnés aléatoirement. Le MRT fournit des informations sur le comportement de la population en matière de transports : possession de véhicules, de permis de conduire et d'abonnements de transports publics, intensité journalière et motifs des déplacements, moyens de transport utilisés. Les résultats fournissent une image détaillée du trafic de personnes en Suisse. Ils servent de référence statistique pour l'élaboration et le contrôle de mesures politiques et constituent des données de base pour des analyses approfondies de l'évolution des transports. 
mobiles comme les caravanes ou les tentes. Cette définition large rejoint celle plus récente d'habitat polytopique (Lussault, 2007). Cependant Odermatt (1990) ne s'est appuyé que sur les résultats du RFP pour son analyse empirique et donc sur une définition plus restreinte de la résidence secondaire.

Enfin, le RFP distingue le domicile économique du domicile civil :

«Une même personne pouvant disposer de plusieurs domiciles, le recensement de 2000 établit comme en 1990 une distinction entre le domicile économique et le domicile civil :

1. Le domicile économique d'une personne se situe dans la commune où elle réside la majeure partie de la semaine, dont elle utilise l'infrastructure et d'où elle part pour se rendre à son lieu de travail ou de formation.

2. Le domicile civil des personnes de nationalité suisse se situe dans la commune où est déposé leur acte d'origine et où elles paient leurs impôts. Pour les ressortissants étrangers, il s'agit de la commune qui leur a délivré leur permis» (OFS, 2004, 2011).

Dans la plupart des cas, le domicile civil et le domicile économique coïncident. Les personnes qui ont deux domiciles distincts sont, par exemple, les pensionnaires d'institutions, les élèves vivant en internat et les personnes qui résident durant la semaine près de leur lieu de travail ou de formation (domicile économique) et qui rentrent chez elles (domicile civil) en fin de semaine. En vertu de l'ordonnance du 13 janvier 1999 sur le $\mathrm{RFP}_{2000}$, la population prise en compte se réfère au domicile économique. Mais la distinction ne correspond pas toujours au vécu. Il y a du reste régulièrement des controverses juridiques sur le domicile fiscal légitime des multilocaux (Kissling, 2010; Duchêne-Lacroix, 2011b; Hosp, 2011 ; Strohm, 2012). La multirésidentialité, comme pratique officielle de deux logements au moins, heurte le paradigme de l'univocité du domicile (container domiciliaire) des autorités territoriales. Les arguments de légitimité se basent alors sur des traces matérielles de pratiques multilocales : domicile du noyau familial, ticket de transport, facture d'électricité, adhésion active à une association locale notamment. Pour beaucoup cela perturbait l'inscription concrète de leur sentiment d'appartenance territoriale. Un grand nombre n'avait pas cultivé de liens particuliers avec Bâle même s'il y percevait un revenu. À notre connaissance, il n'y a pas eu de travaux sur les navettes entre domicile civil et domicile économique. La question sur deux domiciles éventuels a juste servi de filtre pour attribuer un logement «principal» à toutes les personnes y compris dans les cas de birésidentialité8. L'échantillon à $5 \%$

8. «Étant donné l'importance politique et financière de cette notion pour les cantons et les communes, il a paru indispensable de procéder à un ré-examen du concept de domicile utilisé dans les recensements, et ce, d'autant plus qu'à la suite du relevé de 
des données de base des $\mathrm{RFP}_{1970-2000}$, disponible pour la recherche, ne comporte pas les informations nécessaires pour estimer les relations entre ces lieux. Cependant, on remarquera que la question du logement principal est soulevée tout en changeant le terme «domicile économique». Ce sont des critères de fréquence d'habitation qui passe avant les critères juridiques résidentiels. Mais il n'y a plus dans le terme «domicile économique» de hiérarchie par rapport aux autres logements.

Même sans avoir recours aux cas d'étude qui ont pu être discutés pour définir les cas litigieux du RFP ou qui ont été examinés qualitativement dans d'autres recherches (Bonnin, Villanova, 1999), on voit bien que la secondarité a priori d'une résidence par rapport à une autre n'est pas aussi évidente en pratique. Il faut aussi évoquer un autre point : c'est l'usage même du terme résidence secondaire qui évoque la villégiature quand bien même le second domicile serait en ville et pour des raisons professionnelles comme l'a analysé Odermatt (Odermatt, 1990). Les termes d'habiter multilocal, de multirésidentialité ${ }^{9}$, d'habiter polytopique $^{10}$ (Stock, 2006), de domus éclaté11 (Bonnin, 1999) ou de système résidentiel12 (Dureau, 2002), mais aussi plus spécifiquement de domicile économique/domicile civil (RFP), évitent cette hiérarchie ex ante tout en

décembre 1970, les milieux ruraux s'étaient sentis désavantagés par le fait que les navetteurs avaient été attribués à la population de leur lieu de séjour. De même, on n'avait souvent pas compris que le recensement ne tienne pas compte du domicile légal pour attribuer une personne à la population d'une commune. Un groupe de travail mis sur pied par l'Union des Offices Suisses de Statistique (UOSS) a alors été chargé d'étudier ces problèmes. Cependant, le manque de prescriptions légales pour réglementer le séjour des navetteurs hebdomadaires au plan fédéral, c'est-à-dire le risque de ne pas pouvoir déterminer l'effectif de la population selon des principes homogènes ainsi que les difficultés rencontrées lors du relevé des caractéristiques relatives aux pensionnaires d'établissements ont fait pencher la balance en faveur de l'ancien système d'attribution et de relevé. Le Conseil fédéral a adopté cette proposition par l'arrêté du Conseil fédéral du 16 août 1978» (OFS 1981, S.7).

9. Multirésidentialité : Habiter multilocal formel d'une personne ou d'un groupe de personnes, dont le caractère formel peut être attesté entre autre par un nom sur la boite au lettre ou sur une facture d'électricité, un contrat de bail ou la domiciliation administrative à la commune.

10. Habiter polytopique : Ensemble de pratiques de lieux géographiques distincts d'individus mobiles. Le concept d'habiter de Mathis Stock est très extensif, il s'agit de l'ensemble des pratiques des lieux géographiques. L'ajout de «polytopique» permet d'insister sur l'éclatement géographique et la division fonctionnelle des lieux.

11. Domus éclatée : Entité composée du groupe domestique (pouvant être en partie délocalisé), de son espace résidentiel (plusieurs logements), et de l'ensemble de ses ressources (cf. 1999).

12. Système résidentiel : «mode d'habitat articulant plusieurs aires de résidence séparées dans l'espace et occupées différentiellement dans le temps» (Pinson, 1988). 
la permettant ex post selon les besoins de l'analyse et désancre l'ensemble des pratiques de l'image emblématique de la villégiature. Encore faut-il pour être efficace que ce retournement paradigmatique puisse être opérationnalisé dans les enquêtes, ce qui n'est pas clairement le cas en Suisse.

\section{La pratique et les pratiquants}

La disposition de plus d'un logement en propre est un aspect matériel important de l'habiter multilocal. Le logement est une ressource pour la pratique spatiale (ou «ressource d'action spatiale», «raumbezogenes Handlungsvermögen», «spacing capacity to act») et le résultat de la pratique spatiale des acteurs (Rérat, Lees, 2011 ; Duchêne-Lacroix, Schad, 2013). Il est donc un indicateur indirect de la pratique elle-même.

Si on se centre plus directement sur les pratiques multirésidentielles, les données existantes sont (encore) moins étoffées et le cadre d'analyse reste souvent le logement secondaire ou un deuxième logement. Il peut y avoir indexation des questions sur la question filtre de la disposition d'un logement secondaire mais l'informel n'apparait pas (colocation non signalée ${ }^{13}$, nuitées régulières dans un hôtel à proximité du travail, etc.). On peut cerner les populations multirésidentielles dans leur pratique soit par leurs mobilités ${ }^{14}$ (Urry, 2000 ; Hannam et al., 2006), soit par leurs séjours/nuitées sur les lieux/hors les lieux. Mais quelles limites et catégories doit-on établir pour parler d'habiter multilocal?

Et notamment, à partir de quelle fréquence et de quelle relation au logement (fonctionnelle, statut) peut-on attribuer un type de caractère multilocal à des pratiques résidentielles? Ces questions ne peuvent être éclipsées. Pour autant, elles ne peuvent pas non plus être totalement traitées a priori en dehors d'une problématique empirique. En effet, certains terrains de recherche s'intéresseront par exemple aux formes résidentielles, à l'investissement des lieux en portant une grande attention aux résidences en propriété et ouvrant largement l'éventail des durées et fréquences de séjour ; d'autres chercheront à analyser les conséquences de l'hypermobilité sur les offres de transports ou sur la cohésion familiale, etc.

13. En Suisse comme en Allemagne, les habitants d'une commune sont tenus dans un certain délai de s'inscrire sur le registre communal de population.

14. Un questionnement approchant par les mobilités a pu être mené par Vincent Kaufmann sur les hypermobiles et grands voyageurs (Kaufmann et al., 2000). 
Certains cas peuvent entrer sans discussion dans l'ensemble des pratiques multirésidentielles, mais leur étendue doit être davantage précisée. La villégiature ou les navettes hebdomadaires sont par exemple conformes au modèle pour en être même des manifestations des plus typiques. Les navetteurs hebdomadaires (Wochenpendler/Wochenaufenthalter) sont des personnes voyageant chaque semaine d'un de leur domicile à un autre, quels que soient les lieux de ceux-ci. Dans la documentation du $\mathrm{RFP}_{1990}$, on ajoute une fonction particulière au domicile économique où les navetteurs doivent exercer une activité d'au moins une heure (deux populations sont concernées: actifs et écoliers ou étudiants). L'opérationnalisation de la question pose problèmes : combien de jours par semaine faut-il compter dans un et l'autre des logements pour être comptabilisé comme navetteur hebdomadaire? Autre problème, les deux catégories emblématiques sont superposables : un navetteur qui la semaine habite près de son travail et en fin de semaine jardine dans sa maison de campagne pratique la villégiature intermittente («résidence secondaire») et la navette hebdomadaire. Ces catégories ne peuvent donc pas servir en l'état de modalité d'un caractère «habiter multilocal» nominal.

Les transmigrants sont aussi des multilocaux résidentiels. On notera que l'une des définitions les plus connues de la population ne le mentionne pas explicitement :

«Immigrants are understood to be transmigrants when they develop and maintain multiple relations - familial, economic, social, organizational, religious, and political - that span borders. We came to understand that the multiplicity of migrant's involvements in both the home and host society is a central element of transnationalism» (Glick Schiller et al., 1995).

On fera remarquer que les transmigrants peuvent aussi être dans certains cas des navetteurs hebdomadaires dans leur transmigrations (par exemple rencontres de cadres d'entreprise ou de chercheurs entre Berlin et Paris, frontaliers franco-suisses au long-court) ou localement et peuvent utiliser un de leur logement comme villégiature (la maison au pays). Si les domiciles ne sont pas mentionnés dans la définition, en revanche, celle-ci insiste sur les relations sociales, dimension très importante de la multirésidentialité.

Le rapport des personnes aux logements n'est pas seulement celui de la propriété ou de la location. Il peut être aussi, premièrement, fruit des rapports sociaux familiaux ou amicaux. L'accessibilité habituelle ou l'habiter habituel dans un logement et à ces abords se fait dans un réseau social. On peut par exemple le voir dans le cas des enfants de couples séparés (pouvant pour certains être considérés aussi comme des pendu- 
laires hebdomadaires) comme dans celui des jeunes adultes en phase de décohabitation. D'ailleurs, grâce au Panel Suisse TREE auprès de jeunes adultes en transition entre formation et travail, on pourrait obtenir un aperçu des navetteurs hebdomadaires principalement entre un logement propre et un logement parental en fin de semaine. On pourrait ainsi suivre la décohabitation et la mise en couple (ou pas) d'une cohorte sur 7 ans. La question n'a pas encore été traitée statistiquement, elle est en cours d'apurement. Enfin, on peut le retrouver aussi dans les pratiques de prêt d'appartement ou de cohabitation amicale.

Deuxièmement, ces cas de multirésidentialité sont (pensés comme) provisoires, comme transitionnels ou informels et peuvent ainsi passer inaperçus par rapport à un état normal supposé. Le passage à l'âge adulte, la mutation professionnelle ou l'attente de celle-ci, etc. peuvent se traduire par le choix d'une chambre d'hôtel, d'un hébergement amical pour un temps finalement indéfini. Il y a des arrangements avec les situations multilocales en relation avec les structures locales et translocales (Weiske, 2009).

Ces esquisses de réflexions sur le concept d'habiter multilocal rend évident le besoin de redéfinir nos outils d'analyse : de l'habiter, des groupes concernés («ménage», «famille», «entourage»), des liens sociaux, économiques, identitaires aux lieux d'habitation, des bornes spatiotemporelles incluant ou ordonnant les pratiques dans l'ensemble «habiter multilocal». Il est certain que les temporalités font partie des clefs de compréhension des pratiques. Les rythmes sociaux, les rythmes de déplacements et de séjour, mais aussi la distinction entre phases de vie et configuration du ménage sont notamment utiles. Odermatt simplifie en trois phases - formation, activité professionnelle (dont jeunes célibataires, couple sans/avec enfants), retraite (1990). Elles mettent en relief le lien entre résidentialité et positions/charges sociales dans la famille et la société. La fréquence des séjours, la durée des séjours sont des indicateurs utiles sans être pour autant suffisants pour situer les profils de multilocaux. Le niveau pertinent d'échelle des lieux d'habitation est aussi à questionner comme le fait Mathis Stock en proposant le concept d'habiter polytopique (2006). Enfin, un des premiers défis de ce nouveau champ est de proposer un paradigme nouveau tout en s'appuyant sur des données existantes reposant sur des concepts antérieurs. La partie suivante propose une analyse des données disponibles pour la Suisse. 


\section{Estimations quantitatives de l'habiter multilocal}

Les données disponibles les moins éloignées du concept défini se classent selon trois entrées : par le bâti (les logements secondaires), les ménages (birésidentiaux résidant principalement en Suisse) et les pratiques mobiles des habitants multilocaux.

\section{Approche par les logements secondaires}

Le recensement fédéral de la population (RFP) de 2000 dénombrait 419819 logements secondaires, c'est-à-dire «habités temporairement» selon la définition proposée par l'Office fédéral de la Statistique (OFS, 2004). Cela représente un taux de logements secondaires de $11,8 \% 15$ (parmi 3569181 logements recensés en tout) soit 3 points de plus qu'en 1980 (239 446 logements secondaires sur 2722432 logements) (OFS, 1984). C'est-à-dire que la progression du nombre de logements secondaires en 20 ans fut de $75 \%$ tandis que le volume total de logements ne progressait que de $31 \%$. La question n'a pas été exploitée pour l'année 1990 et les données du $\mathrm{RFP}_{2010}$ sont en cours d'apurement avec de plus une autre méthodologie d'enquête.

En 2000, plus de la moitié des logements secondaires (238 000) est située dans et aux alentours des grands centres urbains et non dans les zones touristiques montagneuses. Mais la proportion des logements secondaires des villages de montagne attire l'attention. Certains villages touristiques ont jusqu'à $70 \%$ de résidences secondaires et la majorité en ont plus de $20 \%$ (Sonderegger, 2012). Dans les cinq premières villes suisses (Zürich, Genève, Bâle, Berne et Lausanne), les logements occupés occasionnellement représentent $5 \%$ de l'ensemble des logements (OFS, 2004).

La pression foncière touche autant la campagne touristique que les grands centres urbains. Le poids des résidences secondaires est un problème pour les petits villages de montagne et leur gestion qui agit sur le prix de l'immobilier et accentue le phénomène dit des «lits froids», c'està-dire des logements inoccupés une grande partie de l'année et de l'impression de village fantôme. Le sentiment d'envahissement est accentué par la proportion d'étrangers souhaitant acquérir un logement dans ces régions touristiques contre laquelle des arrêtés furent pris dès les an-

15. Le dernier recensement décennal est en cours. Mais c'est un recensement par les registres de population. 
nées 1950 puis une première loi entra en vigueur en 1984 (Lex Friedrich) assouplie en 1997 (Lex Koller) pour être abrogée en 2007 (abrogation effective en 2010).

Les grandes villes suisses doivent aussi faire face à un manque de logements. Il est courant que les personnes qui ont un logement en propriété ou en location à Zürich préfèrent, en cas de déménagement, louer celuici plutôt que le céder afin, en cas de retour, de se loger facilement. Le nombre élevé de résidences secondaires dans les grands centres urbains est aussi à attribuer à la population étudiante qui souvent se partage entre colocation en semaine et chambre chez les parents le week-end. Cette thèse peut être affermie sur la base de la distribution des birésidentiaux par groupe d'âge que nous allons examiner.

\section{Approche par les birésidentiaux formels habitant en Suisse}

Quittons le comptage des logements secondaires pour celui des ménages ayant un habitat secondaire officiel. Selon les données du MRT 2005 , 8,5\% des ménages en Suisse disposeraient d'une «résidence secondaire»16. Ce qui est surprenant, c'est l'écart avec les données du Panel Suisse des Ménages (PSM) qui établit en 2003 à 15,1\% le nombre de ménages avec résidence secondaire, et ce alors même que la question paraît plus serrée : «Avez-vous une résidence secondaire ?» (PSM) contre «Estce que vous-même ou quelqu'un d'autre dans votre ménage avez une résidence secondaire, qu'il s'agisse d'une maison, d'un appartement ou d'une chambre ?» (MRT). Un regard sur la première vague du PSM donne un niveau similaire de $15,4 \%$ de ménages ayant une résidence secondaire $(\mathrm{N}=5000)$. Pour le MRT, il n'y a pas de comparaison interne possible ; la question sur les résidences secondaires a été introduite en 2005 pour, qui plus est, répondre à des questions de mobilité mais pas d'habitat.

On peut ici supposer un écart dû à la méthode d'enquête. Le PSM est mené chaque année par l'institut de sondage M. I. S. Trend selon la technique de l'interview téléphonique assistée par ordinateur (CATI) et en 3 langues (français, l'allemand et l'italien). Le MRT est aussi conduit sous CATI tous les 5 ans et il a aussi été par le passé mené par MIS (LinkInstitut pour 2005 et 2010). La première vague du Panel a obtenu un taux de réponse de $64 \%$. Le taux de réponse pour le MRT s'élevait à

16. Ce niveau serait en baisse, $7,3 \%$ en 2010 , d'après les premières publications du dernier Microrecensement de 2010 (Kanton Graubünden, 2012). 
environ $65 \%$ en 2005 (MRT Mno0 $_{2}$ : $66 \%$ ). Rien pour l'instant ne permet de définir exactement d'où provient cet écart si ce n'est éventuellement un traitement différent des échantillons plus lourd mais plus fin pour le MRT $\left(\mathrm{N}_{2005}=32000\right)$ que pour le PSM ( $\left.=5000\right)$. Une étude menée par des géographes de Neuchâtel sur l'attractivité des grands centres urbains a montré que $12,4 \%$ et $14,4 \%$ des habitants des logements construits depuis 2001 dans les régions urbaines de Neufchâtel et de ZurichWest disposaient d'un deuxième domicile ${ }^{17}$ (Rérat et al., 2008). L'étude fouillée a analysé une population au «statut socio-économique plus élevé (niveau de qualification, revenu mensuel, statut d'occupation, etc.) que la population totale de ces villes» (Rérat et al., 2008) montrant des résultats similaires à ceux du $\mathrm{PSM}_{2003}$. Or comme nous le verrons ci-dessous, la fréquence de multirésidentialité formelle croît avec le revenu du ménage. Ainsi, le niveau de fréquence de résidence secondaire dans l'enquête de Neuchâtel doit être supérieur au niveau moyen général et non l'inverse. Pour ces raisons et en raison du nombre de ménages interrogés par rapport au PSM, le résultat du MRT devrait être plus fiable.

Une analyse bivariée du profil des birésidentiaux met en lumière en creux des comportements moins formels, par exemple selon l'âge, le niveau du revenu ou le nombre de voitures du ménage.

La fréquence de disposition d'un habitat ${ }^{18}$ secondaire selon l'âge de la personne la plus âgée du ménage est sinusoïdale. Elle est importante au début de l'âge adulte, probablement ici la chambre chez les parents peut être encore choisie comme résidence secondaire (comme ressource pratique aussi). Puis avec l'autonomisation des parents cette possibilité se perd après 40 ans. La fréquence de la birésidentialité s'accroit ensuite jusqu'à un apogée à la classe 60-65. On peut ici y voir une augmentation des ressources directes ou par héritage (logement des parents) avec l'âge. Enfin, le niveau baisse de nouveau pour finir après 75 ans proche de la moyenne générale. Il est possible soit que certains retraités cèdent leur logement secondaire ou principal aux descendants ou le vendent par manque d'usage propre, soit, interprétation générationnelle, que les générations plus anciennes ont eu moins accès financièrement à une résidence secondaire que les générations qui suivirent.

17. La question posée était la suivante : «Avez-vous un deuxième domicile où vous vous rendez régulièrement? Si oui, s'agit-il de votre domicile principal ; votre résidence ou maison de vacances; autre à préciser». On notera des inflexions sur la pratique «visite régulière» - ainsi que l'inversion de hiérarchie de domicile possible. Le deuxième domicile pouvant être le domicile principal.

18. «Habitat» se substitue à «résidence» pour la suite de l’analyse afin de souligner le caractère formel des lieux d'habitation habituels que «résidence» peut évoquer. 
La fréquence des habitats secondaires s'accroit avec le revenu du ménage. Selon les classes de revenu, la fréquence des ménages disposant d'un logement secondaire se situe entre $5,1 \%$ pour les revenus inférieurs à $2000 \mathrm{CHF} / \mathrm{mois}$ et 25,3\% au-delà de $16000 \mathrm{CHF} / \mathrm{mois}\left(\mathrm{MRT}_{2005}\right)$. Il est plus facile d'entretenir un deuxième habitat avec un revenu élevé, cependant une minorité significative de ménages aux revenus le plus bas a encore un logement secondaire. On peut supposer qu'une partie de ces ménages soit constituée de jeunes adultes (par exemple étudiants) aillant la jouissance d'une chambre chez leur parent, ce qu'une étude plus approfondie devrait confirmer.

Figure 1 Proportion de ménage disposant d'au moins un habitat secondaire selon l'âge de la personne la plus âgée du ménage

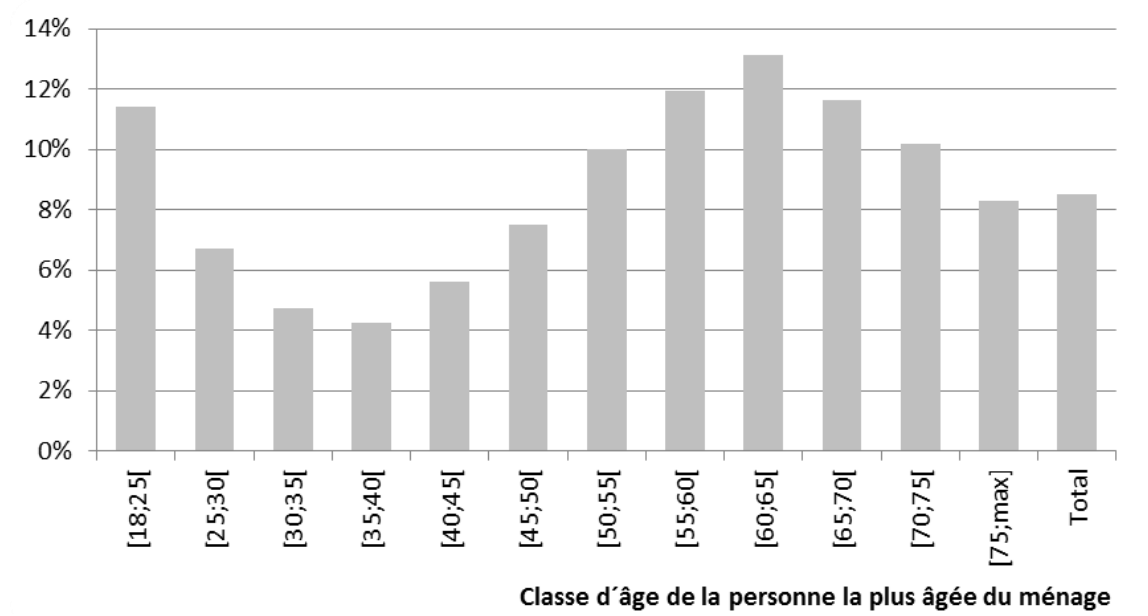

Source : $\mathrm{MRT}_{2005}, \mathbf{N}=\mathbf{3 1} 950$.

Avec le nombre de voitures dans le ménage augmente la fréquence des habitats secondaires. En dehors du besoin d'être mobile pour atteindre les lieux d'habitation, plusieurs effets agissent sur les deux variables comme le niveau de revenu ou le nombre de personnes dans le ménage en âge de conduire. Une étude plus approfondie permettra de déterminer la part de ces motifs et de voir si un effet «voiture» est décelable indépendamment de ces autres variables. D’autres études ont montré que la possession par le ménage de voiture est très dépendante du revenu de celui-ci (ARE, 2006). En outre, les coûts de fréquentes navettes entre les lieux d'habitation sont plus faciles à absorber avec un revenu supérieur. 
FIGURE 2 Proportion de ménage disposant d'au moins un habitat secondaire selon le revenu mensuel du ménage en Francs

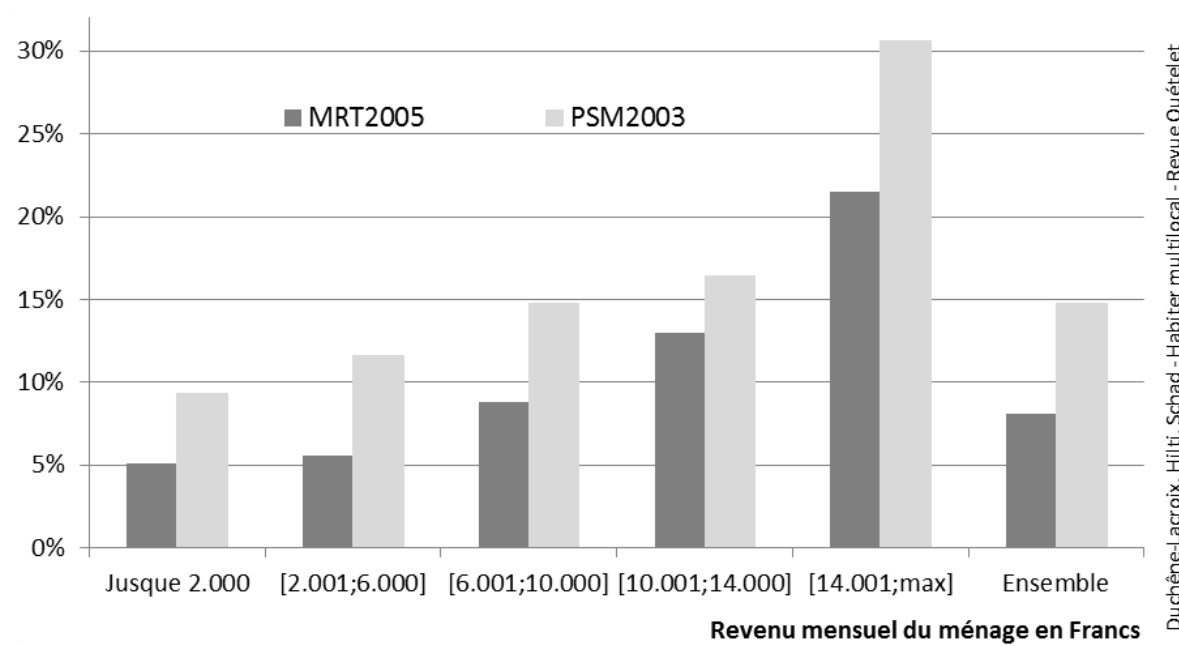

Source : $\mathrm{MRT}_{2005}, \mathrm{~N}=26$ 199, $\mathrm{PSM}_{2003}, \mathrm{~N}=2899$.

FigURE 3 Proportion de ménage disposant d'au moins un habitat secondaire selon le nombre d'automobiles possédées par le ménage

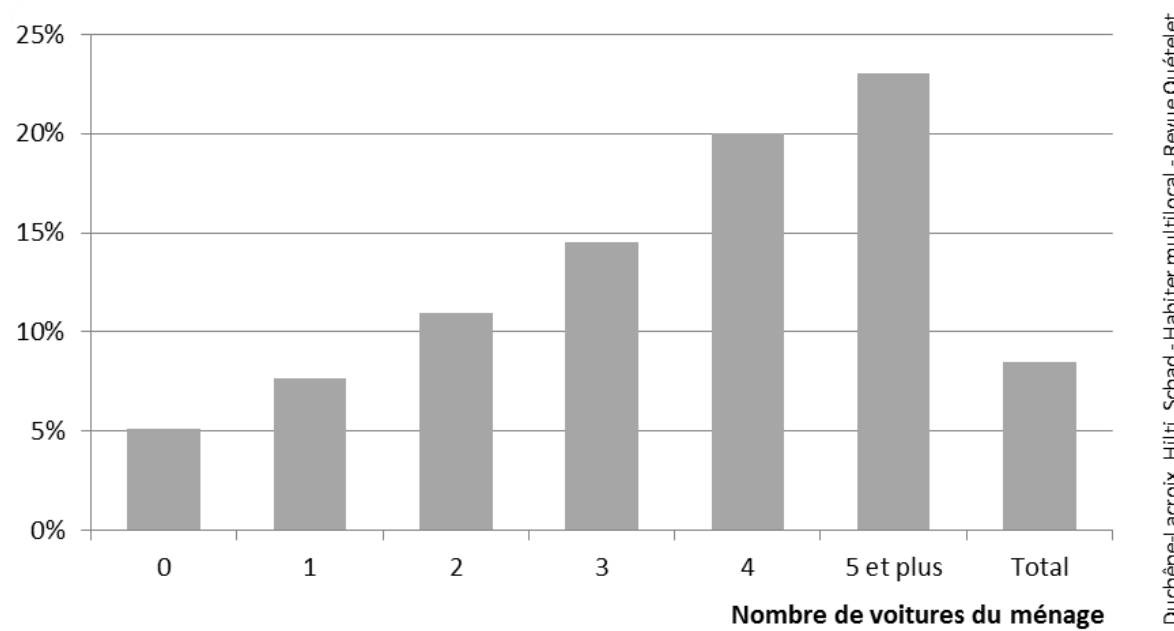

Source: $\mathrm{MRT}_{2005}, \mathrm{~N}=31952$.

Le nombre de ménage avec plus de 5 voitures est faible comme le montre le tableau correspondant en annexe. 


\section{Approche par les pratiques}

L'habiter multilocal se matérialise dans l'habitat, mais ne s'y limite pas. Pour analyser cette pratique, des approches complémentaires sont nécessaires. Nous proposons quatre entrées non pas idéales, mais se basant sur les possibilités existantes, soit : par les usages de l'habiter, par les nuitées hors d'un logement «principal» ou du logement de référence de l'enquête, par les mobilités entre les lieux d'habitation et par l'entretien des rapports sociaux avec l'entourage.

Les habitats du multilocal abritent une variété de pratiques dont la principale est celle de dormir. Toutefois la nécessité de dormir quelque part n'explique pas la multiplication des lieux d'habitation. La question de l'usage principal du deuxième logement est posée dans le microrecensement. Dans $85 \%$ des cas, il est utilisé principalement pour les loisirs $\left(\mathrm{MRT}_{2005}\right)$. Sur les $15 \%$ restant, 10,8 \% des résidences secondaires sont utilisées pour des activités professionnelles ou de formation $(5,1 \%$ pour le travail, 2,4 pour le propre exercice d'une profession, 3,3\% pour la formation). Cela va dans le sens commun. Mais la connotation «villégiature» du terme «résidence secondaire» peut aussi entraîner une surreprésentation des habitations de loisir. En outre, le format de la question ne permettait pas de donner plus d'une réponse, ni de différencier les activités entre membres du ménage. Il peut en outre y avoir un double effet normatif: ces usages de loisirs ou d'activités professionnelles ou étudiantes peuvent être croisés avec leur localisation en Suisse. Sans surprise, les résidences secondaires de vacances sont situées dans les régions touristiques et montagneuses et les résidences professionnelles dans les grands centres urbains (Zürich, Berne, Bâle, ville universitaire) (ARE, 2009). Il serait très utile d'avoir aussi la cartographie des habitats informels selon les types d'usage principaux et secondaires.

Deuxièmement, on peut approcher l'habiter multilocal par les rythmes de séjours et de changement de lieu d'habitation par rapport à un logement de référence (par exemple les pendulaires professionnels «shuttles» ou les pendulaires récrétifs «recrational commuting», les rythmes saisonniers ou irréguliers). Dans une acception large, ces habitants multilocaux rassembleraient les nuiteurs ${ }^{19}$, c'est-à-dire les personnes passant au moins soixante nuitées par an hors du logement de référence de

19. Overnighter en anglais, Übernachter/Übernachtende en allemand. Il n'y a pas encore d'expression française patentée. Découcheur est connoté. Nous proposons simplement nuiteur. 
l'enquête (pas seulement pour des raisons professionnelles définit dans l'enquête «Jobmob» (Huynen et al., 2008)). Sur la base du MRT $2005,2,7 \%$ des personnes interrogées sur leur mobilité ne sont pas parties de leur canton de domicile principal au jour de référence. Elles sont $3,7 \%$ à n'être pas parties de leur commune de domicile principal (Tableau en annexe). Sous-catégorie des nuiteurs, les navetteurs hebdomadaires, qui résident en semaine hors du domicile de référence, représentaient au RFP de 1990 2,4\% de la population résidente (6 873 687) dont 1,7 \% de professionnels (115 494) et 0,7\% d'écoliers ou d'étudiants (48567) (OFS, 1993). Le MRT ne permet pas d'estimer cette population mais celle plus spécifique encore des navetteurs hebdomadaires avec la résidence secondaire. La question était: «combien de jours par semaine utilisez-vous, vous ou une autre personne de votre ménage, la résidence secondaire $x$ ?» Si on prend en compte toutes les réponses supérieures ou égales à une journée par semaine pour l'ensemble des logements secondaires, on obtient seulement $1 \%$ de navetteurs hebdomadaires. On peut être surpris du faible usage non d'une personne mais de l'ensemble des personnes des ménages disposant d'un logement secondaire.

Troisièmement, l'habiter multilocal peut être saisi par les enquêtes sur la mobilité qui distinguent les déplacements avec nuitée. Le MRT dispose d'un module correspondant. Le répondant peut choisir parmi les raisons principales du déplacement la visite d'amis et de connaissances. Des formes d'habiter multilocal pour d'autres motifs ne peuvent toutefois pas être identifiées par cette enquête. De même, des informations complémentaires devraient être demandées comme la fréquence de ces déplacements, le type d'hébergement ou encore les activités pratiquées sur place.

Quatrièmement, l'habiter multilocal formel et informel peut être saisi empiriquement au travers des rapports sociaux localisés qui produisent de l'espace. L'exemple emblématique ici est celui des couples dits noncohabitants (LATs, Living-Apart-Together). Leur multilocalité est largement inaperçue statistiquement car formellement soit chacun a un logement et ne déclare pas le logement du partenaire comme son logement secondaire, soit chacun fait partie d'un même ménage avec un logement principal et un logement secondaire utilisé de façon informelle comme logement principal par l'un des membres. La proportion de couples non-cohabitants est élevée en Europe et atteint $10 \%$ dans plusieurs pays (Duncan, Phillips, 2010) y compris en France selon une enquête française de l'INED sur les liens familiaux (Régnier-Loilier, 2010).

Enfin, les populations cibles (hypermobiles, doubles résidents) sont plus difficiles d'accès que les autres et font habituellement davantage partie 
des non-répondants des enquêtes pour cause d'absence. Seul le RFP, qui doit être exhaustif, permet de dépasser le problème, mais depuis 2010, celui-ci ne pose plus la question.

\section{Bilan et perspectives}

L'habiter multilocal, sa multiplication, est, pour paraphraser Philippe Bonnin (1999), un fait, encore mal connu mais bien réel agissant sur (et agit par) la cohésion familiale, les infrastructures, les normes et le tissu social. Au paradigme ancien des résidences secondaires, l'habiter multilocal ajoute une multitude de pratiques formalisées ou informelles, vécues souvent comme provisoires, mais qui macrosociologiquement persistent tant que les conditions socioéconomiques le permettent (Vincent-Geslin, Kaufmann, 2012). L'habiter multilocal évite ainsi les hiérarchies a priori de résidence (principale, secondaire) et l'image de villégiature des résidences secondaires.

L'habiter multilocal, nouveau concept, est quasi-absent des études statistiques. Les sources suisses montrent des statistiques sur les résidences secondaires, fluctuantes selon les sources et méthodes (de 8,5\% à $15 \%$ de ménages en disposeraient en Suisse entre 2000 et $2005,7,3 \%$ en 2010). L'article a montré que la jouissance d'une habitation secondaire était socialement structurée par l'âge, le revenu du ménage et le nombre de voitures possédées (influencé par le revenu). Des analyses en cours approfondiront la connaissance des effets respectifs des variables d'âge, de genre, de taille de ménage, de capitaux culturels, économiques, sociaux et spatialisés. Les statistiques parcellaires de l'habiter multilocal donnent des fréquences modestes. Les indicateurs de disposition d'autres logements sont des indicateurs indirects des pratiques d'habitation. Nous proposons une approche «directe» et globale des pratiques multilocales.

Dans ces conditions empiriques, on peut se demander si la population concernée est systématiquement sous-évaluée? Des arguments vont dans ce sens. Premièrement, la définition de la population enquêtée et concernée peut être actuellement trop restreinte. Souvent hors-champ des enquêtes, les mineurs de couples séparés sont souvent des multilocaux. Toutes les personnes du ménage d'un multilocal sont aussi concernées par la multilocalité. Deuxièmement, les collectes traditionnelles sous-représentent les profils mobiles qui sont plus fréquemment multihabitants. Plus spécifiquement, la méthode CAPI avec random route 
renforce les difficultés d'entrer en contact avec les multihabitants parce qu'elle demande une présence au domicile de la personne choisie à certaines heures de la journée et la tentation de s'adresser aux personnes présentes peut être grande. La méthode CATI basée sur des bases de téléphones fixes aux domiciles des ménages, qui ne sont plus quasi-exhaustives, doit être complétée par un tirage sur liste de téléphones mobiles; de plus, la population multilocale est moins joignable au domicile que la population monolocale. Dans cette situation, une approche pluriméthode des populations fait sens. Quatrièmement, l'approche par la disposition de logements minore un ensemble de pratiques d'habitation plus informelles. Les individus définissant leurs lieux d'habitation ne vont pas nécessairement penser ni aux habitations hors villégiatures (effet «résidence secondaire») ni aux pratiques informelles, même régulières : la chambre chez les parents ou dans un hôtel habituel ou même un emplacement de camping à l'année. Enfin, étant donné que l'habiter multilocal est un ensemble de pratiques, il serait peut être judicieux de questionner les pratiques spatiales avant de les catégoriser en fonction des caractéristiques résidentielles. C'est ce que l'INED a formulé dans l'enquête Familles et Logement de 2011 qui est en cours d'apurement : «Logez-vous aussi ailleurs habituellement ?» C'est aussi la réflexion que nous avons initiée au sein du réseau international «multilocalité» et qui a été opérationalisée dans une enquête régionale en Allemagne : «Beaucoup de personnes utilisent aujourd'hui d'autres logements à différents endroits pour des séjours intermittents (par exemple logement du partenaire ou logement sur le lieu de travail). Cela vous concerne-t-il ou quelqu'un de votre ménage ?» ${ }^{20}$. Ce type de question insiste sur l'habitude de la pratique et ouvre largement le spectre des lieux d'habitation hors du logement de l'entretien. On pourrait préciser les habitats possibles : «(exemple maison, appartement, chambre, hôtel, mobilhome)». Cinquièmement, des raisons fiscales ou personnelles peuvent conduire les multilocaux réels interrogés à ne pas communiquer la réalité de leur habitation secondaire. Pour diminuer ces cas, il faut notamment insister sur l'objectif scientifique de la recherche sur l'habiter multilocal.

Enfin, la recherche sur l'habiter multilocal est à ces débuts. Elle recèle un fort potentiel tant empirique pour la compréhension de nos sociétés

20. «Viele Menschen nutzen heute zusätzlich weitere Wohnungen an verschiedenen Orten zum zeitweiligen Verbleib (z.B. die Wohnung des Lebenspartners oder eine Wohnung am Arbeitsort). Trifft das auch auf Sie oder eine andere Person in Ihrem Haushalt zu?» Enquête ménage menée en 2011 sur plusieurs régions allemandes par trois instituts: Institut für Landes- und Stadtentwicklungsforschung, Dortmund, Geographisches Institut der Universität Bonn, Leibniz-Institut für Länderkunde (IfL) et financée par la Deutsche Forschungsgemeinschaft (DFG) (www.ils-forschung.de). 
«mobilo-sédentaires» qu'épistémologique car comme nous l'avons vu elle remet en cause les catégories habituelles d'analyses en double container celui des territoires politiques et celui du domicile adminitratif unique des personnes appartenant à un ménage unique.

\section{Références}

ARE (2006), Raumstruktur und Mobilität von Personen. Ergebnisse einer Sonderauswertung des Mikrozensus 2000 zum Verkehrsverhalten (Technischer Arbeitsbericht), Office fédéral du développement territorial, Berne.

ARE (2009), Zweitwohnungen der Schweizer Bevölkerung. Zusatzauswertung des Mikrozensus zum Verkehrsverhalten 2005, Office fédéral du développement territorial/Bundesamt für Raumentwicklung ARE, Berne.

ARSEnAult S. (2009), "Pratiques familiales transnationales», Anthropologie et Sociétés, 33, pp. 211-227.

Baerenholdt J. O. (2007), Coping with Distances: Producing Nordic Atlantic Societies, Berghahn Books, New York Oxford.

BonNin P. (1999), "La domus éclaté», D’une maison l'autre : parcours et mobilités résidentielles, P. Bonnin, R. DE VILLANOVA (eds), Creaphis Éditions, Paris, pp. 19-43.

BonNin P., Villanova R. DE (eds), (1999), D'une maison l'autre : parcours et mobilités résidentielles, Creaphis Éditions, Paris.

Bryceson D. F., Vuorela U. (eds), (2002), The Transnational Family: New European Frontiers and Global Networks, Berg Publishers, Oxford, New York.

Clément C., Bonvalet C. (2005), «Familles recomposées et ancrage résidentiel», Espaces et Sociétés, 79, pp. 120-121.

Conseil fédéral, Département fédéral de l'environnement (2012), Le Conseil fédéral adopte l'ordonnance sur les résidences secondaires [WWW Document], www.admin.ch/aktuell/00089/index.html?lang=fr\&msg-id=45587.

Diminescu D. (2010), "Présentation du numéro Les Migrants connectés», TIC mobilités et migrations, Réseaux 159, pp. 9-13.

DUCHÊNE-LACROIX C. (2007), Archipels transnationaux et agencements identitaires : présences françaises à Berlin, ANRT, Lille.

DuCHÊNE-LACRoIX C. (2011a), "Archipel», Praxen der Unrast: Von der Reiselust zur modernen Mobilität, J. BAduRA, C. DuCHÊNE-LACROIX, F. HEIDENREICH (eds), Lit-Verlag, Berlin-Münster-Wien-Zürich-London, pp. 135-146.

DUCHÊNE-LACROIX C., (2011b), «Résidences secondaires et multirésidentialité à Bâle : problème politique, problème méthodologique», Cahiers de démographie locale, Dynamiques des populations locales 3, (à paraître). 
DUCHÊNE-LACROIX C., SCHAD H. (2013), «Mobilitätskapital, Raumkapital, Räumlichkeitskapital: Ein «Sieg des Ortes über die Zeit» mit welchem raumbezogenen Handlungsvermögen?», Mobilitäten und Immobilitäten, J. Scheiner (ed), Dortmund, (à paraître).

Duncan S., Philuips M. (2010), «People Who Live Apart Together (LATs) - How Different are They?», Sociological Review, 58, pp. 112-134.

DUREAU F. (2002), "Les systèmes résidentiels : concepts et applications», L'accès à la ville. Les mobilités en question, Collection Habitat et sociétés, L'Harmattan, Paris, pp. 355-382.

EMBER M. (1972), "The Conditions Favoring Multilocal Residence», Southwestern Journal of Anthropology, 28, pp. 382-400.

FLORIDA R. L. (2005), Cities and the Creative Class, Routledge, New York \& Abingdon.

Fondation Franz Weber, Helvetia Nostra (2011), Initiative populaire fédérale «Pour en finir avec les constructions envahissantes de résidences secondaires», [WWW Document], Halte aux constructions envahissantes de résidences secondaires, www.zweitwohnungsinitiative.ch/Linitiative.html.

Glick SChiller N., BASCH G. L., Blanc-Szanton C. (1995), «From Immigrant to Transmigrant: Theoretizing Transnational Migration», Anthropological Quartely, 68, pp. 48-63.

Hannam K., Sheller M., URRY J. (2006), «Editorial: Mobilities, Immobilities and Moorings", Mobilities, 1, pp. 1-22.

HıLtı N. (2007), «Nicht daheim und doch zuhause? Über das Phänomen der Multilokalität», Schweizerisches Archiv für Volkskunde, 103, pp. 181-199.

HiLtı N. (2009), «Multilokales Wohnen: Bewegungen und Verortungen», Informationen zur Raumentwicklung, pp. 77-86.

Hiltı N. (2012), Hier - Dort - Dazwischen. Lebenswelten multilokal Wohnender im Spannungsfeld von Bewegung und Verankerung. VS Verlag für Sozialwissenschaften, Stuttgart.

Hosp J. (2011), Kalte Betten in der Stadt, Tagesanzeiger.ch.

Huynen P., Montulet B., Hubert M. (2008), "Survey Design and Methods», Mobile Living Across Europe I. Relevance and Diversity of Job-Related-Spatial Mobility in Six European Countries, N. F. SCHNEIDER, G. MEIL (eds), Budrich, Leverkusen, pp. 47-63.

Kaufmann V., Jemelin C., Joye D. (2000), Entre rupture et activités : vivre les lieux du transport : de la sociologie des usages à l'aménagement des interfaces, Direction du PNR 41, Programme national de recherche, "Transport et environnement, interactions Suisse/Europe», Lausanne.

KesselRING S. (2006), «Pioneering Mobilities: New Patterns of Movement and Motility in a Mobile World», Environment and Planning A 38, pp. 269-279.

KIssuing H. (2010), «Das «kalte Betten» - Problem», Das Magazin, 35, pp. 31-33. 
LE GALL J. (2005), «Familles transnationales : bilan des recherches et nouvelles perspectives», Les Cahiers du Gres, 5, pp. 29-42.

LUSSAUtt M. (2007), L'Homme spatial : la construction sociale de l'espace humain, Seuil, Paris.

Miranda A. (1996), "L'agencement de la multilocalité depuis le lieu d'origine. Le cas de Casalvieri (Sud de l'Italie) et ses migrants», Espaces et sociétés, 80, pp. 271-296.

Odermatt A. (1990), Zweitwohnungen in Städten. Eine Untersuchung über die Zweitwohnungsproblematik in den fünf schweizerischen Grossstädten, Geographisches Institut Univ. Zürich-Irchel, Zürich.

OFS (1984), Recensement fédéral de la population 1980. Logements 1ère partie: conditions de propriété, confort, mode d'occupation, communes (Statistique de la Suisse 706), Office fédéral de la Statistique, Berne.

OFS (1993), Eidgenössische Volkszählung 1990. Räumliche Mobilität, Bundesamt für Statistik, Bern.

OFS (2004), Recensement fédéral de la population 2000. Bâtiments, logements et conditions d'habitation, Office fédéral de la Statistique, Neuchâtel.

OFS (2011), Glossaire [WWW Document], www.media-stat.admin.ch/stat/ haushalte/main/help/help_fr.php.

Perlik M. (2011), "Gentrification alpine : lorsque le village de montagne devient un arrondissement métropolitain", Revue de géographie alpine, (online), 99-1, http:// rga.revues.org/index1385.html.

Petzold K., Weiske C., Zierold D. (2010), «Neue multilokale Haushaltstypen», Unsichere Zeiten: Verhandlungen Des 34. Kongresses Der Deutschen Gesellschaft Für Soziologie in Jena 2008. VS Verlag für Sozialwissenschaften, H.-G. SOEFFNER (ed), Wiebaden.

Pierre M. (2006), "L'invention d'un système bi-résidentiel : un choix de vie pour couple retraité aisé $=$ Retired couples with two homes», Annales de la recherche urbaine, 100, pp. 107-113.

Pinson D. (1988), Du logement pour tous aux maisons en tous genres, Ministère de l'équipement et du logement, Plan construction et architecture.

PrIEs L. (2001), "The Approach of Transnational Social Spaces: Responding to New Configurations of the Social and the Spatial», New Transnational Social Spaces, L. Pries (ed), Routledge.

RÉGNIER-LoILIER A. (2010), Portraits de familles : l'enquête Étude des relations familiales et intergénérationnelles, INED.

Rérat P., Lees L. (2011), «Spatial Capital, Gentrification and Mobility: Evidence from Swiss Core Cities», Transactions of the Institute of British Geographers, 36, pp. 126142. 
Rérat P., Piguet E., Söderström O., Besson R. (2008), Back to the City. Étude de l'évolution démographique et de l'attractivité résidentielle des villes suisses, Institut de Géographie, Université de Neuchâtel, Neuchâtel.

ReUSCHKE D. (2010), Multilokales Wohnen. VS Verlag für Sozialwissenschaften, Wiesbaden.

RoLSHOVEn J. (2004), "Mobilität und Multilokalität als moderne Alltagspraxen», Zweitwohnsitze Und Kulturelle Mobilität, J. ROLSHOVEN, U.GYr (eds),. Zürich, pp. 205-212.

RoLSHOVEN J. (2006), «Woanders daheim. Kulturwissenschaftliche Ansätze zur multilokalen Lebensweise in der Spätmoderne», Zeitschrift für Volkskunde, 102, pp. 179194.

Roth P., Braun U. (2005), Spotlight. Zweit - und Ferienwohnungen in der Schweiz, Credit Suisse, Research Institute.

SCHAD H., DuCHÊNe-LACRoIX C. (2013), "Multilokales Wohnen als hybride Praxis Implikationen der "mobilities studies» und der Akteur-Netzwerk-Theorie», Mobilitäten und Immobilitäten, J. SCHEINER (ed), Dortmund, p. (angenommen).

SCHIER M. (2009), «Räumliche Entgrenzung von Arbeit und Familie. Die Herstellung von Familie unter Bedingungen von Multilokalität», IzR 1/2, pp. 55-66.

SENCÉBÉ Y. (2007), «Individualisme de repli sécuritaire ou d'ouverture affinitaire. La pluralité des ressorts du périurbain", Annales de la recherche urbaine, 102, pp. 5968.

SONDEREGGER R. (2012), Zweitwohnungen im Alpenraum. Entwicklung, aktueller Stand und Perspektiven eines gesellschaftlichen Bedürfnisses.

Sтоск M. (2006), «L'hypothèse de l'habiter poly-topique : pratiquer les lieux géographiques dans les sociétés à individus mobiles», EspacesTemps.net.

SтRонм D. (2012), Viele Zweitwohnungen auch in Städten. Nicht nur die klassischen Tourismusorte haben Probleme mit "kalten Betten», NZZ Online.

StURM G., MeYeR K. (2009), «Was können Melderegister deutscher Großstädte zur Analyse residenzieller Multilokalität beitragen?», IzR 1/2, pp. 15-29.

StURM G., WeISKE C. (eds.), (2009), Multilokales Wohnen, Informationen zur Raumentwicklungen. Bundesamt für Bauwesen und Raumordnung, Bonn.

TARRIUS A. (1992), Les fourmis d'Europe : migrants riches, migrants pauvres et nouvelles villes internationales, L'Harmattan, Paris.

URRY J. (2000), «Mobile sociology», British Journal of Sociology, 51, pp. 185-203.

Vincent-Geslin S., Kaufmann V. (eds.), (2012), Mobilité sans racines : plus loin, plus vite... plus mobiles ?, Descartes \& Cie, Paris.

WEICHHART P. (2009), «Multilokalität - Konzepte, Theoriebezüge und Forschungsfragen», IzR 1/2, pp. 1-14. 
WEISKE C. (2009), "Multilokale Arrangements des Alltags und die kommunalpolitischen Implikationen", Geographie kennt keine Grenzen, B. KÖPPEN, U. MOLTER, I. SCHERM (eds), Chemnitz, pp. 277-289.

Zoul K. (2007), Stabile Gemeinschaften: Transnationale Familien in der Weltgesellschaft, 1, Aufl. ed. Transcript, Bielefeld. 


\section{Annexe statistique}

TABleau 1 Ménage disposant d'au moins un logement secondaire selon l'âge de la personne la plus âgée du ménage

\begin{tabular}{|l|c|c|c|}
\hline $\begin{array}{l}\text { Âge de la personne } \\
\text { la plus âgée du ménage }\end{array}$ & $\begin{array}{c}\text { Avec ou sans habi- } \\
\text { tat } \\
\text { secondaire }\end{array}$ & \multicolumn{2}{|c|}{$\begin{array}{c}\text { Avec habitat secondaire } \\
\text { Paleurs absolues }\end{array}$} \\
\hline $18-24$ & 500 & 57 & $\begin{array}{c}\text { Proportion } \\
\text { des ménages (\%) }\end{array}$ \\
\hline $25-29$ & 1516 & 102 & 11,4 \\
\hline $30-34$ & 2433 & 115 & 6,7 \\
\hline $35-39$ & 3164 & 135 & 4,7 \\
\hline $40-44$ & 3625 & 203 & 4,3 \\
\hline $45-49$ & 3450 & 259 & 7,6 \\
\hline $50-54$ & 3073 & 307 & 10,0 \\
\hline $55-59$ & 3071 & 367 & 12,0 \\
\hline $60-64$ & 2983 & 391 & 13,1 \\
\hline $65-69$ & 2333 & 271 & 11,6 \\
\hline $70-74$ & 1640 & 167 & 10,2 \\
\hline 75 et plus & 4162 & 345 & 8,3 \\
\hline Total & 31950 & 2719 & 8,5 \\
\hline
\end{tabular}

Source : $\mathrm{MRT}_{2005}$; traitement : CDL.

TABLEAu 2 Part des ménages en Suisse ayant au moins un logement secondaire selon le revenu mensuel du ménage ( $\mathrm{CHF}$ )

\begin{tabular}{|c|c|c|c|c|}
\hline \multirow[b]{2}{*}{$\mathrm{MRT}_{2005}$} & \multirow{2}{*}{$\begin{array}{l}\text { Avec ou sans } \\
\text { habitat } \\
\text { secondaire }\end{array}$} & \multirow{2}{*}{$\begin{array}{l}\text { Répartition des } \\
\text { classes } \\
\text { de revenus (\%) }\end{array}$} & \multicolumn{2}{|c|}{ Avec habitat secondaire } \\
\hline & & & $\begin{array}{l}\text { Valeurs } \\
\text { absolues }\end{array}$ & $\begin{array}{c}\text { Proportion des } \\
\text { ménages (\%) }\end{array}$ \\
\hline Jusque de 2000 & 1076 & 4,1 & 55 & 5,1 \\
\hline $2001-6000$ & 12554 & 47,9 & 700 & 5,6 \\
\hline $6001-10000$ & 8764 & 33,5 & 771 & 8,8 \\
\hline $10001-14000$ & 2609 & 10,0 & 339 & 13,0 \\
\hline 14 001-max & 1196 & 4,6 & 257 & 21,5 \\
\hline Ensemble & 26199 & 100,0 & 2122 & 8,1 \\
\hline
\end{tabular}

\begin{tabular}{|c|c|c|c|c|}
\hline \multirow[b]{2}{*}{$\mathrm{PSM}_{2003}$} & \multirow{2}{*}{$\begin{array}{c}\text { Avec ou sans } \\
\text { habitat } \\
\text { secondaire }\end{array}$} & \multirow{2}{*}{$\begin{array}{c}\text { Répartition des } \\
\text { classes de } \\
\text { revenus (\%) }\end{array}$} & \multicolumn{2}{|c|}{ Avec habitat secondaire } \\
\hline & & & $\begin{array}{l}\text { Valeurs } \\
\text { absolues }\end{array}$ & $\begin{array}{c}\text { Proportion des } \\
\text { ménages (\%) }\end{array}$ \\
\hline moins de 2000 & 118 & 4,1 & 11 & 9,3 \\
\hline $2001-6000$ & 1096 & 37,8 & 127 & 11,6 \\
\hline $6001-10000$ & 1056 & 36,4 & 156 & 14,8 \\
\hline $10001-14000$ & 420 & 14,5 & 69 & 16,4 \\
\hline 14 001-max & 209 & 7,2 & 64 & 30,6 \\
\hline Ensemble & 2899 & 100,0 & 427 & 14,7 \\
\hline
\end{tabular}

Source : $\mathrm{MRT}_{2005}$ et $\mathrm{PSM}_{2003}$; traitement : CDL. 
TABleau 3. Proportion de ménage avec au moins un logement secondaire (y compris chambre) selon le nombre d'automobiles possédées par le ménage

\begin{tabular}{|l|r|r|r|}
\hline $\begin{array}{l}\text { Nombre de voitures } \\
\text { dans le ménage }\end{array}$ & $\begin{array}{c}\text { Avec ou sans } \\
\text { habitat } \\
\text { secondaire }\end{array}$ & $\begin{array}{c}\text { Avec habitat secondaire } \\
\text { Valeurs absolues }\end{array}$ & $\begin{array}{c}\text { Proportion } \\
\text { des ménages (\%) }\end{array}$ \\
\hline 0 & 6023 & 307 & 5,1 \\
\hline 1 & 16170 & 1240 & 7,7 \\
\hline 2 & 8031 & 877 & 10,9 \\
\hline 3 & 1310 & 190 & 14,5 \\
\hline 4 & 305 & 61 & 20,0 \\
\hline 5 et + & 113 & 26 & 23,0 \\
\hline Total & 31952 & 2701 & 8,5 \\
\hline
\end{tabular}

Source : $\mathrm{MRT}_{2005}$, traitement : CDL. 